\title{
Influence of Ceramic and Substrate Types on the Microleakage of Aged Porcelain Laminate Veneers
}

This article was published in the following Dove Press journal:

Clinical, Cosmetic and Investigational Dentistry

\author{
Yasir Alnakib (D) \\ Ammar Alsaady $\mathbb{B D}^{2}$ \\ 'Department of conservative Dentistry, \\ Faculty of Dentistry, University of Al- \\ Ameed, Karbala, Iraq; ${ }^{2}$ Department of \\ Conservative Dentistry, College of \\ Dentistry, Mustansiriya University, \\ Baghdad, Iraq
}

Objective: To evaluate the effects of the type of ceramic, and the influence of the type of cervical substrate on the microleakage of aged Porcelain laminate veneers (PLVs).

Materials and Methods: A total of 48 sound human maxillary premolars were divided randomly into two groups $(n=24)$, Group A: lithium disilicate PLVs; Group B: zirconia reinforced lithium silicate PLVs. The groups were further subdivided into four subgroups $(n=12)$ : (A1, B1): finishing line placed in Class V composite filling; (A2, B2): finishing line placed in sound enamel. In subgroups A1 and B1 standardized Class V cavities were prepared and restored with nanocomposite. Standardized PLVs tooth preparation was done for the specimens in all subgroups. Cementation of PLVs was done with a light cured resin cement and specimens were stored in distilled water for 2 weeks. Mechanical load cycling $(45,000$ cycle, $49 \mathrm{~N}$ at $2.5 \mathrm{~Hz})$ and thermocycling procedure $\left(500\right.$ cycles, $\left.5-55^{\circ} \mathrm{C}\right)$ were done. A microleakage test was done with dye penetration ( $2 \%$ methylene blue) and the microleakage percentage was recorded and calculated using a stereomicroscope and ImageJ program.

Results: Means of microleakage percentage of the subgroups were: A1 (6.6075\%), A2 (4.6058\%), B1 (7.3158\%), and B2 (6.105\%), Two-way ANOVA showed a significant effect of ceramic type and cervical composite substrate. According to samples $t$-test, subgroup A2 was significantly lower than A1 and B2, while subgroup B2 was significantly lower than B1. A $P$-value $\leq 0.05$ was considered as statistically significant.

Conclusion: The type of ceramic and the type of substrate both affects PLV microleakage. Lithium disilicate PLVs had significantly lower microleakage compared to zirconia reinforced lithium silicate PLVs. Teeth with cervical composite substrate had a significantly higher microleakage compared to teeth with enamel substrate.

Keywords: dental leakage, dental restoration, dental veneers, VITA Suprinity, IPS e.max CAD

\section{Introduction}

Porcelain laminate veneers (PLVs) as an esthetic treatment modality have seen increased interest worldwide, this is attributed to the combination of excellent esthetics and the strength of dental porcelains with more conservative types of tooth preparation. ${ }^{1}$ PLVs are indicated for the alterations of teeth shape, color, and position. According to a systematic review that evaluated the main clinical performance of glass-ceramic and feldspathic PLVs, the estimated survival for glassceramic PLVs was 94\%, and for feldspathic PLVs was 87\% over a median followup period of 9 years. $^{2}$ The main reasons of failure were fracture and chipping $4 \%$, debonding $2 \%$, severe marginal discoloration $2 \%$, secondary caries $1 \%$, and endodontic failures $2 \%$.
Correspondence: Yasir Alnakib 164/42 Almokhayam District, Karbala, 5600I, Iraq

$\mathrm{Tel}+9647702784555$

Email yasir.alnakib@gmail.com 
Over the last decade, computer aided design/computer aided manufacturing ( $\mathrm{CAD} / \mathrm{CAM})$ of dental restorations has become an established fabrication process, especially for fabrication of PLVs. The PLVs were traditionally fabricated from glass ceramics, this is because of the excellent optical properties of these materials which mimics the shade and translucency of natural dentition. Glass ceramics have evolved over the years in their compositions, physical properties, and processing techniques. Initially feldspathic porcelains had no crystal phase, which isthen evolved to better leucite crystal phase and later lithium disilicate crystal phase Ceramics (L2S). ${ }^{3}$ Recently, a new material was introduced, zirconia-reinforced lithium silicate ceramic (ZLS), with the idea that zirconia could act as a crystal phase nuclei center that can reinforce the glass ceramics material. ${ }^{4}$ This material was created with the hope of uniting the physical properties of polycrystalline ceramics with the esthetic excellence of the glass-ceramics in a CAD/CAM monolithic restoration, however, this material is relatively new and there is little published evidence on its performance.

The use of PLVs with dentinal or composite filling margins has been subjected to controversy. ${ }^{5-7}$ Although researchers suggested that the veneers should be extended to achieve an intra-enamel margin, ${ }^{8}$ this is not always the case in clinical practice. According to a clinical study, $60 \%$ of laminate veneers have been reported to cross over existing composite restorations. ${ }^{9}$

PLVs marginal discoloration has been reported with patient dissatisfaction in clinical studies. ${ }^{2,10,11}$ It hold the proof of marginal defects, partial debonding, and microleakage. ${ }^{12}$ There is a suggestion that the resin cements shrinkage layer can generate internal stress, causing microcrack formation, ${ }^{13}$ this can be accelerated by mechanical load which further propagates the crack marginally and results in microleakage and possible future fracture. Another suggestion is the differences in the coefficient of thermal expansion (CTE) of bonded surfaces (the enamel, the resin cement, the ceramic, and the composite), this difference in behavior of the surfaces under the oral thermal cycles may cause a marginal opening and ultimately microleakage. ${ }^{8}$

Little information is available in the literature on the effects of ceramic materials on the microleakage of PLVs. Also, there is little evidence on the influence of class $\mathrm{V}$ composite filling as bonding substrate on the microleakage of PLVs. The aim of the study is to evaluate the effects of ceramic materials and cervical composite substrate on the microleakage of aged PLVs.

\section{Materials and Methods}

Forty-eight human maxillary first premolar were selected for the study. Teeth were extracted as a part of an orthodontic treatment plan in the clinics of the Department of Orthodontics, College of Dentistry, Mustansiriya University and all patients signed a consent form. The research was approved by the scientific and ethical research committee, College of Dentistry, Mustansiriya University.

All teeth were caries free and carefully examined with light transillumination (Diagnostic LED Attachment, Radii-Plus, SDI, Australia) to detect any crack. Teeth were cleaned using a fluoride-free pumice with prophylaxis rubber cup (Produits Dentaires S.A, Switzerland) and then were stored in $0.1 \%$ thymol solution for 1 week. During all the following steps of the study, the specimens were stored in distilled water.

The specimens were divided randomly into two groups $(n=24)$ according to the type of ceramic material used, Group A: lithium disilicate PLVs (IPS e.max CAD, A1 HT, C14, Ivoclar/Vivadent, Germany); Group B: zirconia reinforced lithium silicate PLVs (VITA Suprinity PC, A1 HT, LS14, Vita Zahnfabrick, Germany). The groups were further subdivided into four subgroups $(\mathrm{n}=12)$ : (A1, B1): cervical finishing line placed in class $\mathrm{V}$ composite filling (Filtek Z350 XT, 3M ESPE, Germany); (A2, B2): finishing line placed in sound enamel.

\section{Simulation of Periodontal Ligament}

Bone support and the periodontal ligament are important for the mechanisms of stress distribution of teeth. The root surfaces were dipped into molten dipping wax (GEO Dip, Renfert, Germany) up to $2 \mathrm{~mm}$ below the cementoenamel junction (CEJ), ${ }^{14}$ resulting in a wax layer of $0.2-0.3 \mathrm{~mm}$ thickness that was ensured by measuring the width of the root from the three facing points before and after dipping using digital caliper (InSize, Austria) (Figure 1A and B). The teeth were mounted individually in a custom-made mold made of rubber silicon material $(20 \times 20 \times 25 \mathrm{~mm})$ with pink cold cure acrylic (Paladur, Kulzer GmbH, Germany) using dental surveyor (Paraline, Dentaurum GmbH, Germany) to ensure vertical positioning of each tooth inside the mold. At the first polymerization sign, each tooth was removed from acrylic block, then wax removed, then A-silicone light body impression material (Elite $\mathrm{HD}+$, Zhermack SpA, Italy) was injected and the tooth was reinserted. A standardized silicone layer of $0.2-0.3 \mathrm{~mm}$ that simulated periodontal ligament was thus created, taking the thickness of the wax layer. 


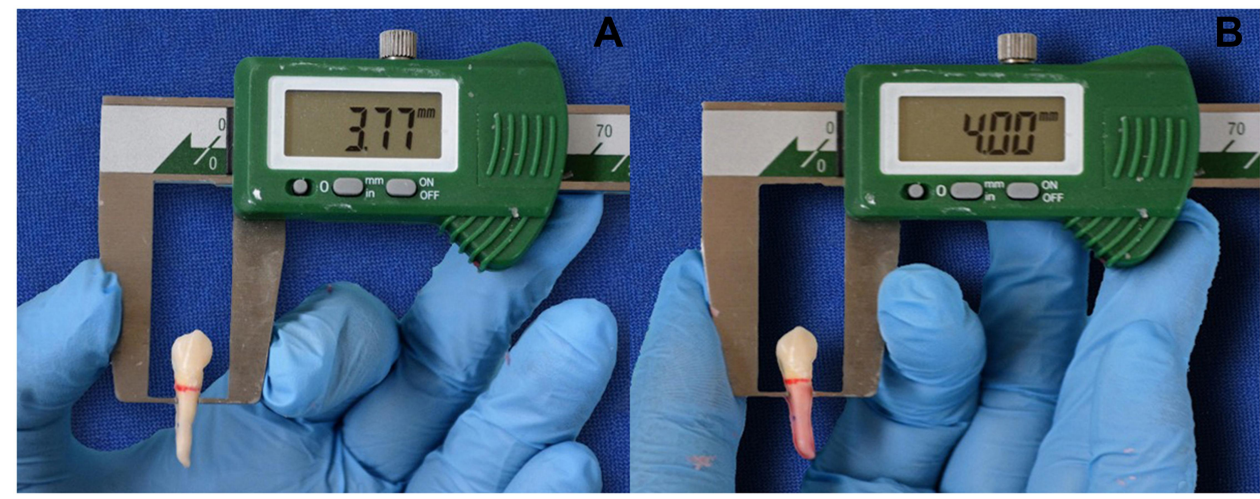

Figure I (A) Measuring the width of the root before wax dipping. (B) Measuring the width of the root after wax dipping.

\section{Preparation of Cervical Composite Substrate}

All specimens of A1 and B1 subgroups received a standardized class $\mathrm{V}$ cavity on the buccal surface. Initially, Class V cavity preparation template was made by using the square edge diamond wheel bur (ISO no. 806314043 524 040, NTI-Kahla GmbH, Germany) with high speed water-cooled hand piece (Kavo Dental GmbH, Germany) that was fixed to the vertical arm of modified dental surveyor to standardize the cavity preparation perpendicular to the long axis of the tooth. Then, Tungsten carbide fissure bur no. 256 (Komet, Germany) was used to standardize the cavity depth and correct the cavity floor. Color marker (Stabilo, China) and digital caliper (InSize, Austria) were used to mark the bur to provide a visual reference at $1.5 \mathrm{~mm}$ depth.

All Class V cavities were prepared $1 \mathrm{~mm}$ above the cementoenamel junction in order to keep the restoration within enamel boundaries. ${ }^{15,16}$ The dimensions of the prepared cavities were: occluso-gingivally $(2 \mathrm{~mm})$ and axial depth $(1.5 \mathrm{~mm})$. The excess color marks were wiped with a cotton pellet dipped in alcohol.

Class V cavities acid were etched with $35 \%$ phosphoric acid etchant gel (Scotchbond ${ }^{\mathrm{TM}}$ Universal Etchant, 3M ESPE, Germany) following manufacturer's instructions, then washed and bloated with cotton pellet. A 5th generation bonding agent (Adper Single Bond 2 Adhesive, 3M ESPE, USA) was applied and light cured (Radii-Plus, SDI, Australia) for 40 seconds. A restorative procedure was done with nano-filled composite (Filtek Z350 XT, 3M ESPE, Germany) in two horizontal layers and light cured (Radii-Plus, SDI, Australia) for 20 seconds for each layer. The composite filling was finished and polished with a finishing polishing kit (Super-Snap Kit, SHOFU INC., Japan).

\section{PLVs Preparation}

For standardization purposes, all of the specimens were prepared by the same operator under $4 \mathrm{x}$ magnification (ZEISS EyeMag Pro S, ZEISS Medical Technology, Germany). Standardized preparations were done for all the teeth using a ceramic veneer system preparation bur set (Keramik-veneers.de, Komet, Germany). At first, a silicon mold was constructed with putty condensation silicone (Ormadent Putty with Ormactivator Gel, Major Prodotti Dentari Spa, Italy) to provide visual reference during tooth preparation (Figure 2A). The outline of the preparation was painted on the tooth with water proof color marker (Stabilo, China) to provide a visual reference for the preparation area. The preparation was $1.5 \mathrm{~mm}$ above CEJ, Buccal cusp $1.5 \mathrm{~mm}$ bucco-palatally and $1.5 \mathrm{~mm}$ occlusocervically. The facial reduction was $0.4 \mathrm{~mm}$ at the cervical third and $0.5 \mathrm{~mm}$ at the middle and occlusal thirds with an occlusal butt-joint preparation (Figure 2B).

At first, a custom made impression tray was fabricated by a pressure moulding machine (Biostar, Scheu-Dental $\mathrm{GmbH}$, Germany). The custom-made trays were perforated with dimensions of 20x20x25 mm. The final impressions for the teeth in all the groups were taken with addition silicone impression material (Elite $\mathrm{HD}+$, Zhermack SpA, Italy) in a two-stage putty wash technique and poured with type IV dental stone (elite model, Zhermack SpA, Italy).

The PLVs were designed with (inLab SW, Sirona Dental Systems, Bensheim, Germany), then milled with a 5-axis milling machine (CEREC inLab MC XL, Sirona Dental Systems, Bensheim, Germany). PLVs were fired in a ceramic firing furnace (Programat CS, Ivoclar Vivadent/ technical, Liechtenstein, Germany) at $840^{\circ} \mathrm{C}$ with vacuum according to the manufacturer's instructions. 


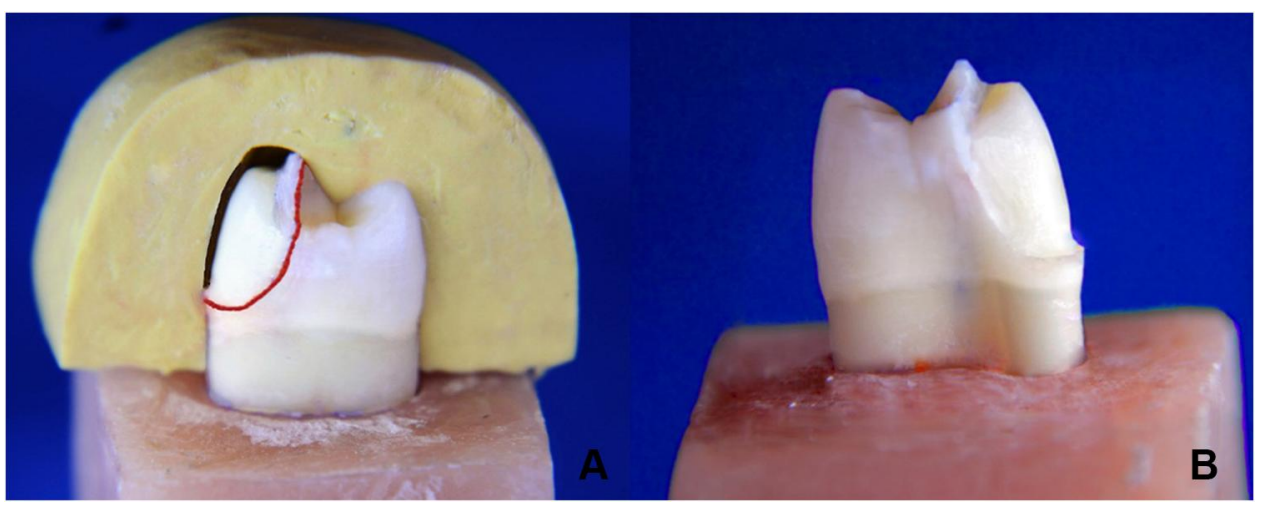

Figure 2 (A) Silicon index for visual reference during preparation. (B) Final tooth preparation.

In groups A1 and A2 with composite substrate, composite surfaces were sandblasted to facilitate the bonding procedure. Sandblasting was done with $29 \mu \mathrm{m}$ alumina oxide $\left(\mathrm{Al}_{2} \mathrm{O}_{3}\right)$ particles for 10 seconds with a sandblasting machine (AquaCare, Velopex, UK). ${ }^{17}$ A custom-made shield was made of additional silicon (Zhermack SpA, Italy), this custom shield contains an opening only over the area of composite restoration to prevent the detrimental effects of sandblasting of tooth structure.

Teeth preparations of all subgroup were etched with $35 \%$ phosphoric acid etchant gel (Scotchbond ${ }^{\mathrm{TM}}$ Universal Etchant, 3M ESPE, Germany) for 20 seconds, rinsed for 20 seconds, and then the excess water was gently air dried for 5 seconds. Immediately after drying, two consecutive coats of 5th generation bonding agent (Single Bond 2 Adhesive, 3M ESPE, USA) were applied to the etched tooth surface for 15 seconds with gentle agitation using a fully saturated brush, then was gently air thinned for 5 seconds to evaporate the solvents and left without curing. ${ }^{18,19}$

The internal surfaces of fabricated PLVs of all groups were etched by using 5\% hydrofluoric acid gel (IPS ceramic etching gel, 3M ESPE, Germany) for 20 seconds according to the manufacturer's instructions. The veneers were then washed thoroughly with air/water spray for 30 seconds and air dried. The veneers were silanated by application of ceramic primer (RelyX ceramic primer, $3 \mathrm{M}$ ESPE, USA) to the internal surface of the veneer and left to dry for 1 minute. One coat of 5th generation bonding agent (Single Bond 2 Adhesive, 3M ESPE, USA) was applied to the internal surface of the veneer and also left without curing.

A small increment of translucent shade light cured resin cement (RelyX Veneer cement, 3M ESPE, USA) was dispensed directly from the syringe onto the internal surface of the veneers. The veneers were seated in place using gentle pressure with placement instrument with an adhesive tip (Optrastick, Ivoclar/Vivadent, Germany), then light cured (Radii-Plus, SDI, Australia) for 40 seconds. The margins were finished and polished with a finishing and polishing kit (Super-Snap Kit, SHOFU INC., Japan). Then, the specimens were stored at $37^{\circ} \mathrm{C}$ in distilled water for 2 weeks.

\section{Simulation of Clinical Situation (Ageing)}

In an attempt to simulate the clinical situation (eg, mastication and oral cavity environment), all the specimens were submitted to mechanical load cycling and thermocycling procedures. A custom-made device was used for the load cycling procedure (Figure 3A). The specimens were subjected to 50,000 cycles of $49 \mathrm{~N}$ at a frequency of $2.5 \mathrm{~Hz}$. The specimens were kept moist during the procedure by using a cannula attached with a distilled water container, this cannula was constantly dripping distilled water on the specimens (Figure 3B). The thermocycling procedure was done using a custom made automatic thermocycling device. The specimens underwent 500 water cycles between $5^{\circ} \mathrm{C}$ and $55^{\circ} \mathrm{C}$, with a dwell time of at least 30 seconds according to International Standardization Organization specifications (ISO/TS 11405:2015). Then, all the specimens were air-dried.

\section{Measurements of Microleakage}

The specimens were immersed in $2 \%$ methylene blue dye in a container for 48 hours at $37^{\circ} \mathrm{C}$. Then, the specimen's crowns were blocked with clear cold cure acrylic (Paladur, Kulzer GmbH, Germany) with a custom-made circular mold (15 $\mathrm{mm}$ in diameter) and sectioned bucco-palatally at the center into two pieces using a microtome (MT-4 


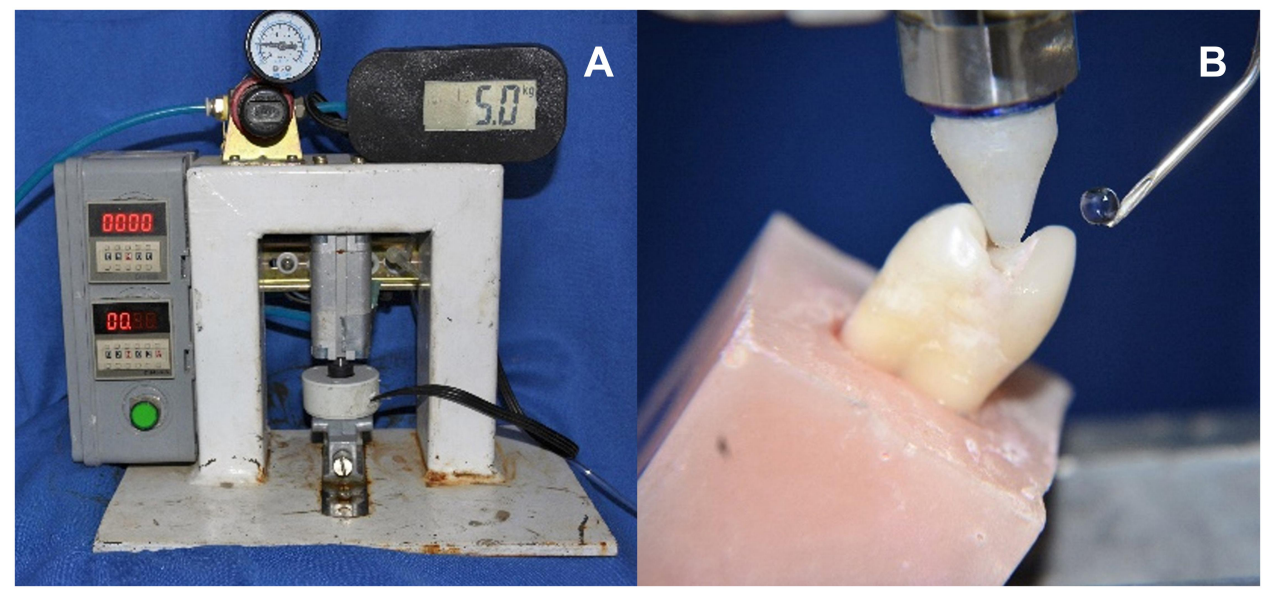

Figure 3 (A) Custom made cyclic loading device. (B) Cycling loading tip in contact with the tooth.

Diamond cut-off saw, USA) with a disk thickness of 0.35 $\mathrm{mm}$ cutting at high speed with water coolant.

The presence of microleakage was confirmed by the visualization of a blue dye extent into the adhesive interface (occlusal and cervical) with a stereomicroscope (Meiji Techno Co. Ltd, Japan). Microleakage measurement is done by using an image processing program (ImageJ software, National Institutes of Health, https:// imagej.nih.gov). Measurement calibrations were done by using a millimeter graph paper as a guide to measure the length in micrometers $(\mathrm{Mm})$ (Figure 4A). At first, the total measurements of the adhesive interface were recorded (Figure 4B), then occlusal, cervical microleakage measurements were recorded (Figure $4 \mathrm{C}$ and D).

The following mathematical formula was used to calculate the microleakage percentage:

Microleakage $\%=[$ occlusal reading $(\mathrm{Mm})+$ Cervical reading $(\mathrm{Mm}) /$ Total measurement of adhesive interface $(\mathrm{Mm})] \times 100 \%$ (The mean of both halves of each specimen is taken as a record).
The workflow of the current research is illustrated in Figure 5.

\section{Statistical Methods}

Statistical analysis was performed by using IBM SPSS Statistics (Statistical Package for Social Science, version 24.0; IBM Corp., Armonk, NY, USA) to calculate descriptive statistics and inferential statistics. Initially, checking for distribution of data by statistical method was done by using both Kolmogorov-Smirnov and Shapiro-Wilk test. Analysis of variance (Two-way ANOVA) was performed as we wanted to evaluate the effect of the two factors (type of ceramic and type of substrate) on the microleakage of aged PLVs. Separate analyses were used in each condition separately (independent sample $t$-test) was used. $P$-value $\leq 0.05$ was considered as statistically significant. $P$-value $\leq 0.01$ was considered as statistically significant. $P$-value $>0.05$ was considered as statistically non-significant.

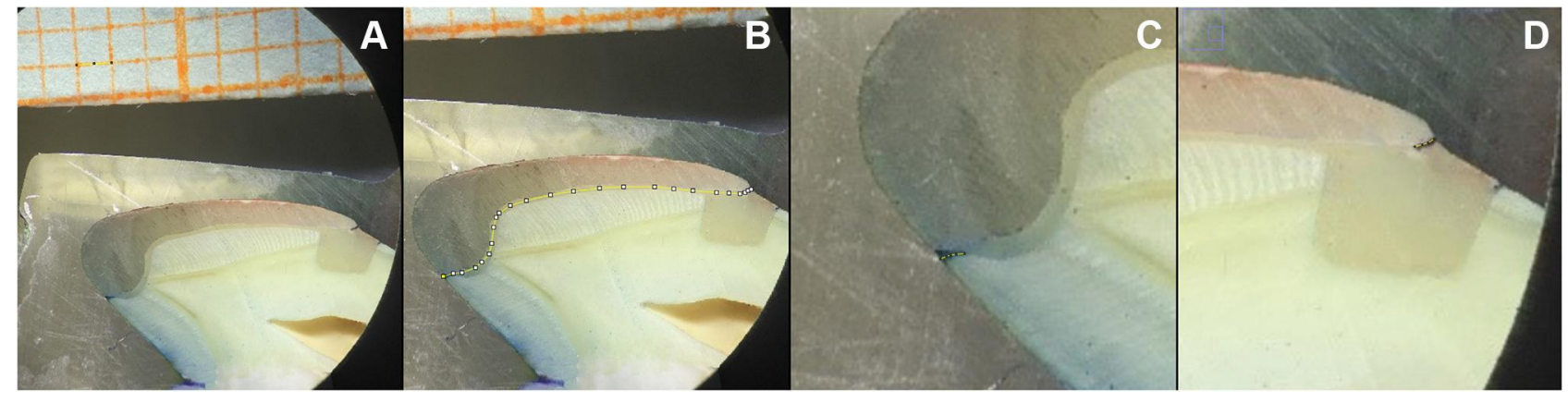

Figure 4 (A) Length measurement calibration. (B) Measurement of total adhesive interface (Mm). (C) Occlusal microleakage (Mm). (D) Cervical microleakage (Mm). 


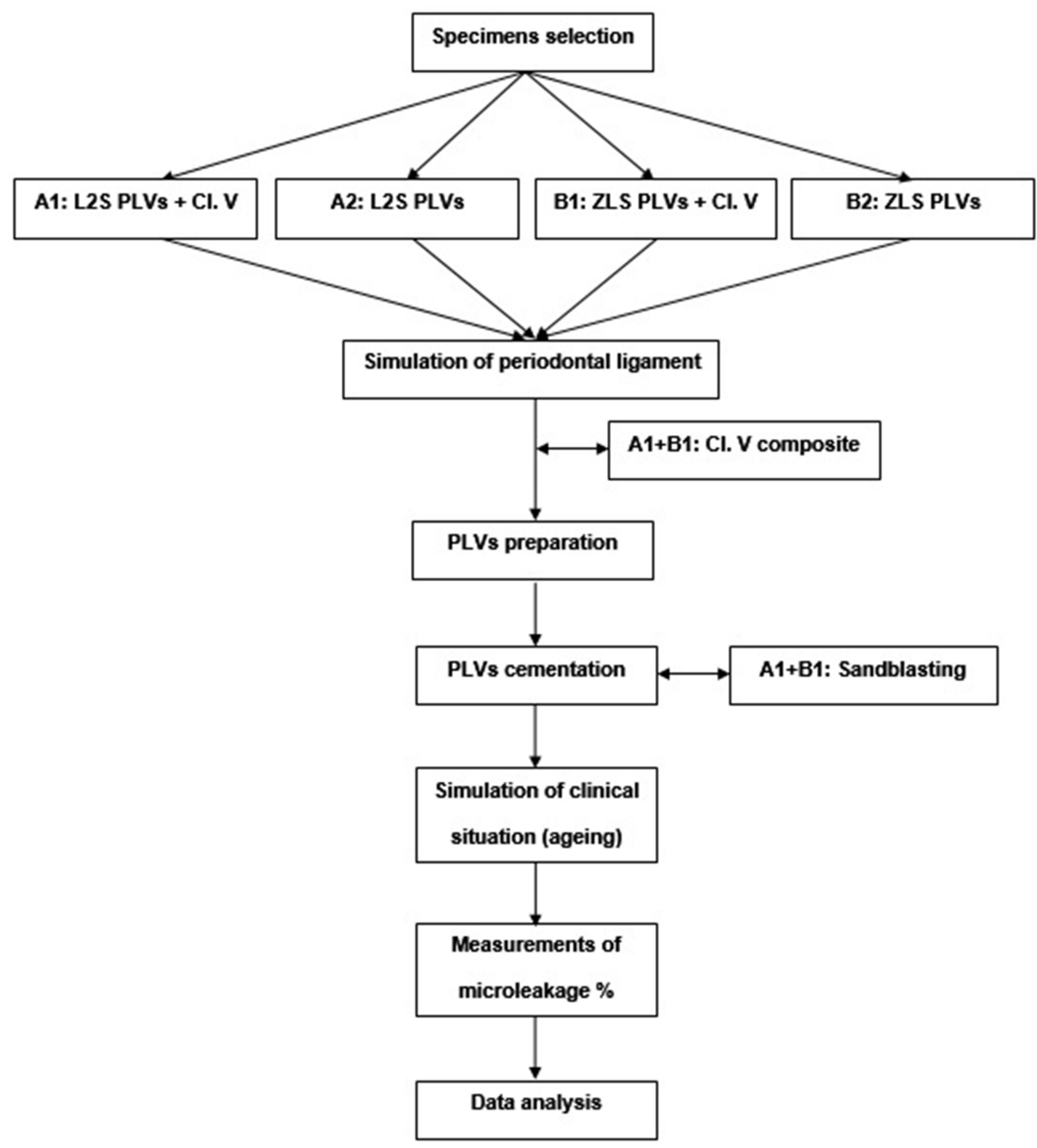

Figure 5 Flowchart of the research protocol workflow.

\section{Results}

The results of this study showed that the lowest mean of microleakage percentage was recorded for subgroup A2, followed by subgroup B2 and subgroup A1, while the highest mean value was recorded by group B1. Kolmoyrov-Smirnov and Shapiro-Wilk test were used to test the distribution of data in statistical methods. According to the results of both tests, normal data distribution was assumed (Table 1).

The two-way ANOVA test indicated the significant effect of the type of ceramic. Similarly, the type of substrate had a highly significant effect. However, the interaction of the effects between those two factors had a non-significant effect on the microleakage percentage (Table 2).

According to samples $t$-tests, the PLVs groups restored with L2S ceramic (A1 and A2) showed highly significant effects of presence of cervical composite substrate on the
Table I Descriptive Statistics (Mean, Standard Deviation of Microleakage Percentage) and Normality of Distribution Tests by Statistical Methods

\begin{tabular}{|l|l|l|l|l|l|}
\hline Subgroup & $\mathbf{n}$ & $\overline{\mathbf{X}}$ & SD & K-S & S-W \\
\hline AI & 12 & 6.6075 & 1.32594 & 0.200 & $0.48 \mathrm{I}$ \\
A2 & 12 & 4.6058 & 1.5091 & 0.200 & 0.447 \\
BI & 12 & 7.3158 & 1.38713 & 0.186 & 0.224 \\
B2 & 12 & 6.105 & 1.456 & 0.200 & 0.999 \\
\hline
\end{tabular}

Abbreviations: $n$, number of specimens; $\bar{X}$, mean; SD, standard deviation; $K-S$, Kolmogorov-Smirnov test; S-W, Shapiro-Wilk test.

increase of microleakage percentage as compared to enamel substrate. Similarly, PLVs groups restored with ZLS (B1 and B2) showed the same significant effect. Within the PLVs groups with cervical composite substrate (A1 and B1), there was a non-significant effect between the two types of ceramics. On the other hand, within the 
Table 2 Two-Way ANOVA Test of Mean Value of Microleakage Percentage of the Ceramic Type Related and Type of Substrate

\begin{tabular}{|l|l|l|l|l|l|}
\hline Source & SS & DF & MS & F & P \\
\hline Ceramic & 14.62 & $\mathrm{I}$ & 14.62 & 7.23 & 0.0101 \\
Substrate & 30.96 & $\mathrm{I}$ & 30.96 & 15.31 & 0.0003 \\
Ceramic * substate & 1.88 & $\mathrm{I}$ & 1.88 & 0.93 & 0.34 \\
Error & 89 & 44 & 2.02 & & \\
Total & 136.46 & 47 & & & \\
\hline
\end{tabular}

Abbreviations: SS, sum of squares; DF, degree of freedom; MS, mean square; F, Fratio; $P$, probability value.

PLVs groups with enamel substrate (A2 and B2), L2S PLVs had significantly lower microleakage percentages compared to ZLS PLVs (Table 3).

\section{Discussion}

Although the ideal setting for the experimental study of dental materials and restorations is the oral cavity, clinical trials are time-consuming and not always cost-effective.20,21 To test materials and restorations in vitro, the test conditions should match the oral environment as closely as possible. $^{22}$ Therefore, periodontal simulation, thermal cycling, and mechanical load cycling were used in the present study. Human teeth were used in this study because they have unique properties, such as bonding characteristics, elasticity, strength, thermal conductivity, ion transfer in dentinal tubules, and enamel thickness. ${ }^{23}$

Bone support and the periodontal ligament are important for the mechanisms of stress distribution of teeth.14,24,25 When load is applied, periodontal fibers are compressed, the tooth dislodges slightly, and there is bone distortion in the direction of the root movement. ${ }^{26}$ The mechanical response of periodontal ligament to external stress is non-linear and viscous, ${ }^{27}$ which is similar to the characteristics of elastomeric impression materials. In this study, the simulation of periodontal ligament is done by creating a layer of $0.2-03 \mathrm{~mm}$ of silicon impression material between the root and the acrylic block. ${ }^{14,28}$

Table 3 Independent Samples $t$-Tests of the Four Study Groups

\begin{tabular}{|l|l|l|}
\hline Source & $\mathbf{T}$ & $\boldsymbol{P}$ \\
\hline $\mathrm{A} 1 \times \mathrm{A} 2$ & 3.45176 & $0.002(\mathrm{HS})$ \\
$\mathrm{B} 1 \times \mathrm{B} 2$ & -2.0828 & $0.049(\mathrm{~S})$ \\
$\mathrm{A}|\times B|$ & $-1.2787 \mathrm{I}$ & $0.214(\mathrm{NS})$ \\
$\mathrm{A} 2 \times \mathrm{B} 2$ & 2.47334 & $0.0215(\mathrm{~S})$ \\
\hline
\end{tabular}

Abbreviations: $\mathrm{T}$, t-value; $P$, probability value.
When placing the margins of a porcelain veneer on an existing composite, it is important to consider that weak bonding may lead to microleakage and fracture. Microleakage could result from a difference in the flexure and thermal expansions of the tooth and the ceramic or resin composite. ${ }^{1,8,10}$ In order to avoid weak bond at the area of existing composite, alumina oxide sandblasting was done to the composite surface at the time of cementation. $^{29}$

Measurement of microleakage was done by measuring the incisal and cervical microleakage in $\mathrm{Mm}$, then dividing them by the total bonded surface of the veneer and multiplying by $100 \%$ to yield the microleakage percentage. All the measurements were done digitally by computer software. ${ }^{18}$ This method of microleakage measurement is considered more objective and precise with less chance of bias compared to the scoring method which depends on the subjective decision of the observer.

According to the results of the two-way ANOVA test, a significant effect was found for the type of ceramic restoration on microleakage percentage of porcelain veneers. This finding disagrees with Zaimoglu and Karaagaclioglu, ${ }^{30}$ who concluded that the porcelain material had no significant effect on the microleakage of porcelain veneers. This may be attributed to the different ceramics used, different restoration fabrication methods, and different methods of record of the microleakage data.

According to the results of the samples $t$-test, the L2S veneers group A2 had a significantly lower microleakage percentage mean than the ZLS group B2. The same finding was noted when comparing between subgroups A1 and B1 (although non-significant), with the highest microleakage percentage recorded for ZLS group B1. There were no previous studies comparing microleakage of both ceramic materials.

The coefficient of thermal expansion (CTE) has been suggested as an important factor that influences microleakage, ${ }^{31-33}$ it is influenced by the composition of the restorative material. A greater difference in the CTE between tooth and restorative material leads to the generation of excessive stresses with temperature fluctuation that may result in micro-cracks that propagate along the bonded interface, causing a gap to form and eventually microleakage. Lower CTE of L2S ceramic $\left(\mathrm{CTE}=10.25 \times 10^{-6} \mathrm{~K}^{-1}\right)$ compared to $\mathrm{ZLS}$ ceramic $\left(\mathrm{CTE}=11.6 \times 10^{-6} \mathrm{~K}^{-1}\right)$ may be the cause of the reported lower microleakage percentage of L2S veneers which translate into better performance during the thermal stressing of the material. ${ }^{34}$ 
On the other hand, one of the methods to reduce microleakage of dental restorative materials reported in the literature is the use of a material with a lower modulus of elasticity (MOE). ${ }^{35,36}$ According to Elsaka and Elnaghy,${ }^{37} \mathrm{~L} 2 \mathrm{~S}$ ceramic had a significantly lower modulus of elasticity (60.61 GPa) compared to ZLS ceramic (70.44 GPa). The combination of lower MOE and lower strength of L2S ceramic translate into higher resiliency compared to ZLS ceramic. ${ }^{38}$ This results in a better elastic buffer and compensation for resin cement shrinkage stress, which is another explanation of the lower microleakage percentage mean of $\mathrm{L} 2 \mathrm{~S}$ veneers.

This study showed a highly significant effect for the cervical composite substrate on the microleakage percentage of porcelain veneers. This finding agrees with Sadighpour et $\mathrm{al}^{7}{ }^{7}$ who concluded that ceramic veneers with class 4 composite filling had significantly higher microleakage compared to controls. The result of the study also agree with Lacy et al, ${ }^{39}$ who demonstrated the complete leakage between the GIC restoration and the porcelain veneers. "However, microleakage records was lower in our study, this may be because of the adhesive behavior of different filling material and/or surface treatment.

According to results of the samples $t$-test, cervical composite substrate group A1 had a significantly higher microleakage percentage mean compared to enamel substrate group A2. The same significant effect is seen when comparing group B1 and group B2, with the first being significantly higher than the latter. These findings agree with Metz et al, who concluded that composite materials as finish lines that interact with resin cements and ceramic crown had significantly higher microleakage compared to controls. $^{40}$

The increased microleakage percentage of the cervical composite substrate groups (A1 and B1) may be attributed to the flexural difference between the tooth structure and ceramic materials which could cause gap formation and increase microleakage. ${ }^{7}$ Another cause may be due to the difference in coefficient of thermal expansion among the tooth structure, composite filling and ceramic veneer which reveal the different behavior of the various materials during thermocycling and load cycling procedures which may cause a larger gap and increased microleakage.

The recorded microleakage data of this study was consistent with other similar studies which used the microleakage percentage as a record method. ${ }^{18,41,42}$ There was no acceptable microleakage rank for porcelain veneers, comparing against the acceptable microleakage score of ceramic crowns.

Further investigations are needed to study the microleakage between indirect restoration and composite substrate, possibly with various surface treatment, different bonding procedures, or different luting cements, since there is a lack of such research in the current literature.

\section{Conclusion}

Within the limitation of this study, it was concluded that:

- The type of ceramic material and the presence of class $V$ filling both as a bonding substrate had significant effects on microleakage percentage of porcelain laminate veneers.

- L2S veneers had significantly lower microleakage compared to ZLS veneers.

- PLVs on teeth with class V composite filling within the cervical finishing line had a highly significant higher microleakage compared to PLVs on teeth without class V composite filling.

- The two types of ceramic veneers when bonded to teeth with class $\mathrm{V}$ composite filling did not have a significant difference in microleakage between them.

\section{Disclosure}

The authors report no conflicts of interest in this work.

\section{References}

1. Peumans M, Van Meerbeek B, Lambrechts P, Vanherle G. Porcelain veneers: a review of the literature. J Dent. 2000;28(3):163-177. doi:10.1016/S0300-5712(99)00066-4

2. Morimoto S, Albanesi RB, Sesma N, Agra CM, Braga MM. Main clinical outcomes of feldspathic porcelain and glass-ceramic laminate veneers: a systematic review and meta-analysis of survival and complication rates. Int J Prosthodont. 2016;29(1):38-49. doi:10.11607/ ijp. 4315

3. Höland W, Apel E, Van't Hoen C, Rheinberger CS. Studies of crystal phase formations in high-strength lithium disilicate glass-ceramics. $J$ Non Cryst Solids. 2006;352(38-39):4041-4050.

4. Krüger S, Deubener J, Ritzberger C, Höland WJ. Nucleation kinetics of lithium metasilicate in $\mathrm{Z} \mathrm{r}$ O 2-bearing lithium disilicate glasses for dental application. Int J Appl Glass Sci. 2013;4(1):9-19.

5. Gresnigt MM, Ozcan M, Kalk W, Galhano G. Effect of static and cyclic loading on ceramic laminate veneers adhered to teeth with and without aged composite restorations. J Adhes Dent. 2011;13(6):569577. doi:10.3290/j.jad.a21742

6. Calamia JR, Calamia CS. Porcelain laminate veneers: reasons for 25 years of success. Dent Clin North Am. 2007;51(2):399-417, ix. doi:10.1016/j.cden.2007.03.008

7. Sadighpour L, Geramipanah F, Allahyari S, Fallahi Sichani B, Kharazi Fard MJ. In vitro evaluation of the fracture resistance and microleakage of porcelain laminate veneers bonded to teeth with composite fillings after cyclic loading. $J$ Adv Prosthodont. 2014;6(4):278-284. doi:10.4047/jap.2014.6.4.278

8. Chun Y-HP, Raffelt C, Pfeiffer H, et al. Restoring strength of incisors with veneers and full ceramic crowns. J Adhes Dent. 2010;12(1):45. 
9. Karlsson S, Landahl I, Stegersjo G, Milleding P. A clinical evaluation of ceramic laminate veneers. Int J Prosthodont. 1992;5(5):447-451.

10. Peumans M, De Munck J, Fieuws S, Lambrechts P, Vanherle G, Van Meerbeek B. A prospective ten-year clinical trial of porcelain veneers. $J$ Adhes Dent. 2004;6(1):65-76.

11. Beier US, Kapferer I, Burtscher D, Dumfahrt H. Clinical performance of porcelain laminate veneers for up to 20 years. Int $J$ Prosthodont. 2012;25(1):79-85.

12. Dennison J, Sarrett D. Prediction and diagnosis of clinical outcomes affecting restoration margins. J Oral Rehabil. 2012;39(4):301-318. doi:10.1111/j.1365-2842.2011.02267.x

13. Magne P, Kwon K-R, Belser UC, Hodges JS, Douglas WH. Crack propensity of porcelain laminate veneers: a simulated operatory evaluation. J Prosthet Dent. 1999;81(3):327-334. doi:10.1016/S00223913(99)70277-5

14. Soares CJ, Pizi ECG, Fonseca RB, Martins LRM. Influence of root embedment material and periodontal ligament simulation on fracture resistance tests. Braz Oral Res. 2005;19(1):11-16. doi:10.1590/ S1806-83242005000100003

15. Majeed MA. Microleakage evaluation of a silorane-based and methacrylate-based packable and nanofill posterior composites (in vitro comparative study). Tikrit J Dent Sci. 2012;1:19-26.

16. Irie M, Tjandrawinata R, Suzuki K. Effect of delayed polishing periods on interfacial gap formation of class V restorations. Oper Dent. 2003;28(5):552-559.

17. Hickel R, Brüshaver K, Ilie NJDM. Repair of restorations-criteria for decision making and clinical recommendations. Dent Mater. 2013;29 (1):28-50. doi:10.1016/j.dental.2012.07.006

18. Ibarra G, Johnson GH, Geurtsen W, Vargas MAJDM. Microleakage of porcelain veneer restorations bonded to enamel and dentin with a new self-adhesive resin-based dental cement. Dent Mater. 2007;23 (2):218-225. doi:10.1016/j.dental.2006.01.013

19. Almeida JR, Schmitt GU, Kaizer MR, Boscato N, Moraes RR. Resinbased luting agents and color stability of bonded ceramic veneers. $J$ Prosthet Dent. 2015;114(2):272-277. doi:10.1016/j. prosdent.2015.01.008

20. Stappert CF, Ozden U, Gerds T, Strub JR. Longevity and failure load of ceramic veneers with different preparation designs after exposure to masticatory simulation. $J$ Prosthet Dent. 2005;94(2):132-139. doi:10.1016/j.prosdent.2005.05.023

21. Ozturk E, Bolay S, Hickel R, Ilie N. Shear bond strength of porcelain laminate veneers to enamel, dentine and enamel-dentine complex bonded with different adhesive luting systems. J Dent. 2013;41 (2):97-105. doi:10.1016/j.jdent.2012.04.005

22. Bayne S. Dental restorations for oral rehabilitation-testing of laboratory properties versus clinical performance for clinical decision making. $J$ Oral Rehabil. 2007;34(12):921-932. doi:10.1111/j.13652842.2007.01812.x

23. Rueggeberg F. Substrate for adhesion testing to tooth structureReview of the literature: a report of the ASC MD156 task group on test methods for the adhesion of restorative materials Accredited standards committee MD156 for dental materials and devices. Dent Mater. 1991;7(1):2-10. doi:10.1016/0109-5641(91)90017-S

24. Burke F, Wilson N, Watts D. The effect of cavity wall taper on fracture resistance of teeth restored with resin composite inlays. Oper Dent. 1992;18(6):230-236.

25. Rees J. An investigation into the importance of the periodontal ligament and alveolar bone as supporting structures in finite element studies. J Oral Rehabil. 2001;28(5):425-432. doi:10.1046/j.13652842.2001.00686.x
26. Picton D, Davies W. Dimensional changes in the periodontal membrane of monkeys (Macaca irus) due to horizontal thrusts applied to the teeth. Arch Oral Biol. 1967;12(12):1635-1643. doi:10.1016 0003-9969(67)90197-5

27. Pini M, Wiskott H, Scherrer S, Botsis J, Belser U. Mechanical characterization of bovine periodontal ligament. J Periodontal Res. 2002;37(4):237-244. doi:10.1034/j.1600-0765.2002.00344.x

28. Yaroub M, Hameed MR. Evaluation of marginal gap at the composite/enamel interface in Class II composite resin restoration by SEM after thermal and mechanical load cycling (An in vitro comparative study). J Baghdad Coll Dent. 2014;26(4):63-70. doi:10.12816/ 0015256

29. Nassoohi N, Kazemi H, Sadaghiani M, Mansouri M, Rakhshan V. Effects of three surface conditioning techniques on repair bond strength of nanohybrid and nanofilled composites. Dent Res $J$ (Isfahan). 2015;12(6):554-561. doi:10.4103/1735-3327.170575

30. Zaimoglu A, Karaagaclioglu L. Influence of porcelain material and composite luting resin on microleakage of porcelain laminate veneers. J Oral Rehabil. 1992;19(4):319-327. doi:10.1111/j.13652842.1992.tb01574.x

31. Gale M, Darvell B. Thermal cycling procedures for laboratory testing of dental restorations. J Dent. 1999;27(2):89-99. doi:10.1016/S03005712(98)00037-2

32. Fasbinder DJ. Restorative material options for CAD/CAM restorations. Compendium. 2002;23(10):911-922.

33. Tan CL, Santini A. Marginal microleakage around class V cavities restored with glass ceramic inserts of different coefficients of thermal expansion. J Clin Dent. 2005;16(1):26-31.

34. Wendler M, Belli R, Lohbauer UJDM. Factors influencing development of residual stresses during crystallization firing in a novel lithium silicate glass-ceramic. Dent Mater. 2019;35(6):871-882. doi:10.1016/j.dental.2019.03.002

35. Fabianelli A, Pollington S, Davidson CL, Cagidiaco MC, Goracci C. The relevance of microleakage studies. Int Dent SA. 2007;9(3):6474 .

36. Kemp-Scholte CM, Davidson CL. Marginal integrity related to bond strength and strain capacity of composite resin restorative systems. $J$ Prosthet Dent. 1990;64(6):658-664. doi:10.1016/0022-3913(90) 90291-J

37. Elsaka SE, Elnaghy AM. Mechanical properties of zirconia reinforced lithium silicate glass-ceramic. Dent Mater. 2016;32:908-914. doi:10.1016/j.dental.2016.03.013

38. Sakaguchi RL, Powers JM. Craig's Restorative Dental Materials, Chapter Four: Fundamental of Materials Science. Vol. 13. Elsevier Health Sciences; 2012.

39. Lacy AM, Wada C, Du W, Watanabe L. In vitro microleakage at the gingival margin of porcelain and resin veneers. $J$ Prosthet Dent. 1992;67(1):7-10. doi:10.1016/0022-3913(92)90038-C

40. Metz M, Durski M, Chou J, Crim G, Harris B, Lin W. Microleakage of lithium disilicate crown margins finished on direct restorative materials. Oper Dent. 2016;41(5):552-562. doi:10.2341/15-225-L

41. Maleknejad F, Moosavi H, Shahriari R, Sarabi N, Shayankhah T. The effect of different adhesive types and curing methods on microleakage and the marginal adaptation of composite veneers. $J$ Contemp Dent Pract. 2009;10(3):18-26. doi:10.5005/jcdp-10-3-18

42. Faus-Matoses I, Solá-Ruiz F. Dental preparation with sonic vs highspeed finishing: analysis of microleakage in bonded veneer restorations. J Adhes Dent. 2014;16(1):29-34. doi:10.3290/j.jad.a30754 


\section{Publish your work in this journal}

Clinical, Cosmetic and Investigational Dentistry is an international, peer-reviewed, open access, online journal focusing on the latest clinical and experimental research in dentistry with specific emphasis on cosmetic interventions. Innovative developments in dental materials, techniques and devices that improve outcomes and patient satisfaction and preference will be highlighted. The manuscript management system is completely online and includes a very quick and fair peer-review system, which is all easy to use. Visit http://www.dovepress.com/testimonials.php to read real quotes from published authors. 